\title{
THE BOEING DOLBLE SUBHARMONIC ELECTRON INJECTOR - PERFORMANCE MEASUREMENTS
}

J. L. Adamski, W. J. Gallagher, R. C. Kennedy,

D. R. Shoffstall, E. L. Tyson, and A. D. Yeremian

Boeing Aerospace Company, P.0. Box 3999, Seatt1e, WA 98124

\section{Summary}

A two stage subharmonic injector has been installed and tested on the Boeing $S$ band linac. The injector is designed as a prototype front end for a high voltage linac for free electron laser research. 1 This accelerator will require long macropulse trains of widely spaced high current micropulses.

Single micropulse output beams of $1-2 \mathrm{nC}, 10$ ps width, $1 \%$ full width energy spread and normalized emittance of $\varepsilon_{\mathrm{n}}=\beta \gamma \pi r \phi=0.01 \mathrm{~cm}-\mathrm{rad}$ have been measured. The data are in good agreement with model predictions. 2

\section{Physical Description}

The subharmonic injector, figure 1, comprises a $5 \mathrm{~A}, 60-100 \mathrm{kV}$ triode electron gun, two standing wave cavity prebunchers and a fundamental frequency traveling wave tapered phase velocity buncher. The first prebunching cavity operates at $119 \mathrm{MHz}$, the 24th subharmonic of the accelerator frequency; the second operates at $476 \mathrm{MHz}$, the 6 th subharmonic. Both cavities are fabricated in stainless steel to minimize beam induced cavity voltage during the intended long pulse operation.

A "pepper pot" emittance measurement, a beam viewing screen and current toroid occupy the space between the prebuncher cavities. A full solenoidal magnetic field provides radial containment and focusing of the electron beam. A tapered collimator in the last drift section limits beam size and entry angle at the buncher. The tapered phase buncher and the first short constant gradient section provide acceleration to 10 MeV. These sections are independently phased to provide optimum bunching. A $16 \mathrm{MW}$, dual-output klystron (Thomson - CSE 2097) powers the sections.

\section{Bunching Design}

The electron source is a ARCo/RPC Model 12 gun modified to our design which uses a substantially reduced active area at the cathode. Simulation calculations, using the SLAC ETP code, ${ }^{3}$ predicted a factor of in improvement in emittance, alnng with a factor of 3 reduction in current, for the small cathode Model 12B. The ETP calculation predicted $5.1 \mathrm{~A}$ at $60 \mathrm{kV}$ operating voltage with an emittance of $2.2 \times 10^{-3} \pi \mathrm{m}_{\mathrm{o}} \mathrm{c}-\mathrm{cm}(0.007$ (m-rad).
A hard tube amplifier circuit in the gun deck provides the $1 \mathrm{kV}$ cathode drive pulse for a $5 \mathrm{~A}$ with $3 \mathrm{~ns}$ FWHM. The cathode pulse circuitry is phase locked to the master, $119 \mathrm{MHz}$, oscillator. The drive electronics can be repetitively pulsed at rates up to $19.8 \mathrm{MHz}$.

The 3 ns gun pulse covers roughly \pm 60 degrees of phase of the first prebuncher cycle time. The electron beam is then compressed on the relatively linear portion of the sinusoidal waveform and we are able to take advantage of nearly the full gap voltage. If all works as planned the bunch fills \pm 60 degrees again in the second prebuncher. The baseline design ORBIT ${ }^{4}, 5$ code analysis of the injector was for $2.5 \mathrm{nC}$ input and operation of the two prebunchers at $30 \mathrm{kV}$ and $20 \mathrm{kV}$ gap voltage. The code results predicted $90 \%$ of the charge, $2.2 \mathrm{nC}$, would be focused to 90 degrees of fundamental phase at the entry to the traveling wave section. Additional bunching in this waveguide predicted an output pulse of $15 \mathrm{ps}$ with the equivalent peak micropulse current of $170 \mathrm{~A}$.

\section{Magnetic Optics}

The magnetic optics design is fully solenoidal with a counter coil at the gun output for fine tuning of the transition between the gun and the first drift. ETP, and an in-house magnetic optics code, LENSING, were used to adjust the 12 separate excitation circuits to fit a Brillouin flow magnetic field profile. The beam radius is tapered from $1 \mathrm{~cm}$ at the gun to $0.2 \mathrm{~cm}$ at the entrance to the buncher.

\section{Performance Data}

The gun emittance and emittance growth due to the $24 / 1$ subharmonic buncher were measured by a multiple hole collimator (pepper pot) and a fluorescent target, $40 \mathrm{~cm}$ downstream. In the constant axial field $-B_{Z}$ (evidenced by no overall magnification of the collimator patcern at the screen), a walst of radius $r_{0}$ is set at the collimator. If the size of the beamlets. from the collimator holes is dominated by rotation in the axial field, then the individual collimator hole image radius, $r^{\prime}$, is a function of $\phi$, the beam far-field angle, and the solenoid field. The normalized emittance can be estimated as follows:

$$
\varepsilon_{\mathrm{n}}=\beta \gamma \pi \mathrm{r} \phi=\pi \mathrm{r}_{\mathrm{o}} \mathrm{m}_{\mathrm{o}} \mathrm{c}^{\mathrm{B}} \mathrm{B} \mathrm{r}^{\prime}
$$

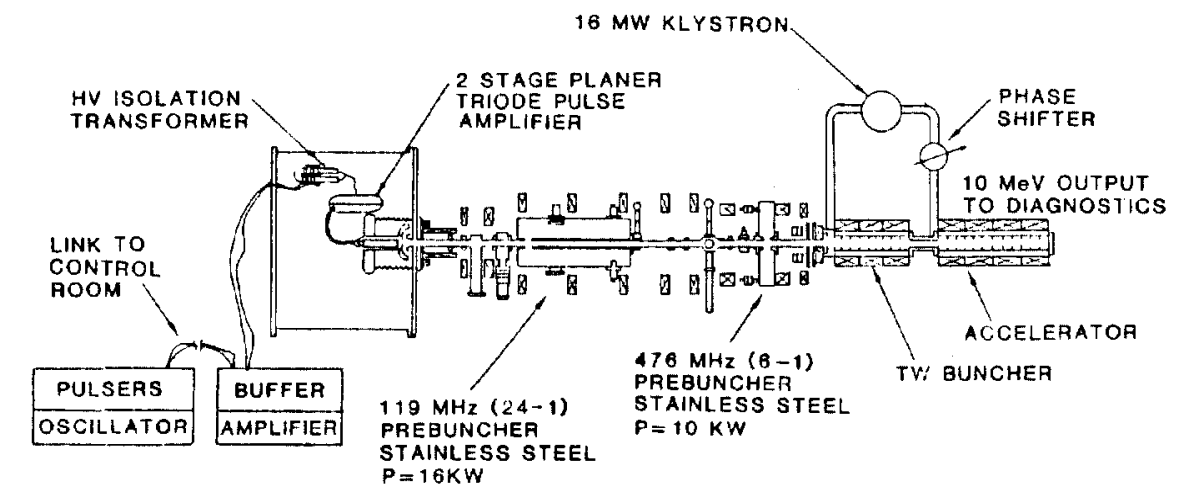

Figure 1 Double Subharmonic Injector

0018-9499/85/1000-2994\$01.00@ 1985 IEEE 
The resolution of emittance data is limited by the size of the holes in the pepper pot. The diameter of the holes is $0.5 \mathrm{~mm}$, which limits the minimum resolvable emittance to $0.003 \mathrm{~cm}-\mathrm{rad}$. The emittance measured for a 3 A current with a 5 -ns (FWHM) pulse width was $0.01 \mathrm{~cm}$-rad. Retaking the measurement with the $24 / 1$ subharmonic buncher turned on did not increase the emittance. A schematic of the measurement technique and a photograph of the fluorescent screen image are shown in figure 2 .
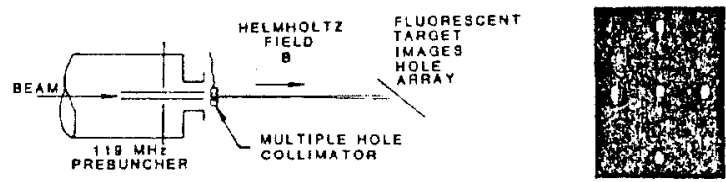

F1gure 2 Eniltance Measurement for Gun and First Prebuncher

The injector output at $10 \mathrm{MeV}$ was fully characterized. The data described in the paragraphs following are simultaneous measurements.

A micropulse diagnostics and tuning station was installed on the straight-ahead beamline. Figure 3 illustrates this station.

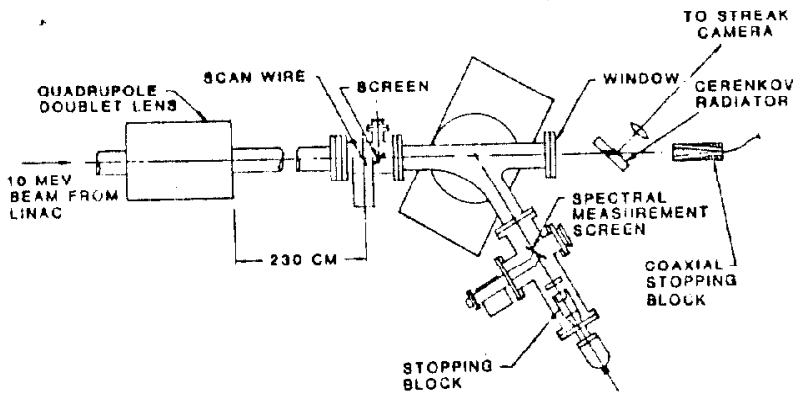

Figure 3 Micropulse Diagnostic Station

Micropulse charge was measured using a $50 \Omega$ coaxial stopping block located directly outside the foil window. The charge was cabled through $150 \mathrm{ft}$ of $7 / 8$-in Styroflex cable and displayed on a Tektronix 7104 oscilloscope (1-GHz bandwidth at $3 \mathrm{~dB})$. A photograph of the data is presented in figure 4 . The total charge contained in the first spike of the waveform (with a 2.3 A height and a $1-n s$ base) is $1.15 \mathrm{nC}$. Because the time interval between s-band micropulses is 350 ps, more than one micropulse could make up the measured 1-ns pulse. To clarify the time structure, a direct measurement of the micropulse width was made by observation of Cerenkov light from a quartz radiator placed in the beam path. The measurement instrument was an Hammatsu 0979 Temporaldisperser (streak camera).

The streak camera data in figure 4, at low time resolution, spans several $\mathrm{RF}$ cycle times clearly demonstrating the single bucket loading. Streak data at full resolution (nominally 7 ps FWHM for a delta function light input, and 2 ps pixel size) is used to measure the macropulse width, figure 5.

The data display of figure 5 shows a measured pulse width of 12 ps FWHM. The actual pulse width, from an rms combination of the measurement and the camera delta function response, is estimated to be $10+2$ ps. This pulse width together with the $1.15 \mathrm{nC}$ yields an average micropulse current of $115 \mathrm{~A}$.

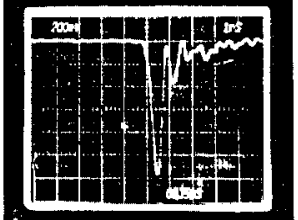
ZOVIOIV INSIOIV $\frac{115 V}{50 \Omega} \times$, NS BASE $\times 1 / 2=1,16 \mathrm{NCOUL}$

Figure 4 Micropulse Charge Measurement

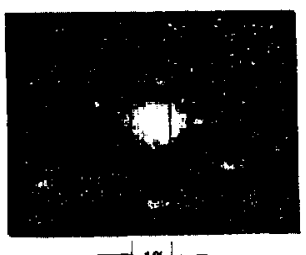

ENERTO

- PHOTO OF BEAM ON SPECTROQR PLANE SHOWg nominal 1\% FllL WIOTH ENERQY SPREAD
SOR FAFADAY CUP CURAEN

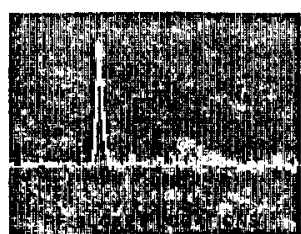

STREAK CAMERA IRACE SHOW THAT ALL CHABGE IS IN ONE FF

\section{- gtreak camera minimum} AESOLUTION Q PQ

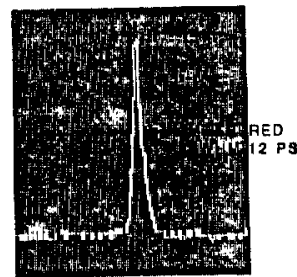

- Streak camea measlifement OF MICAOPIJLSE WIOTH IS

10.2 PS. CONSIS TENT WITH I\$

FULL WIDTH ENEROY SPAEAD
Figure 5 Micropulse Width and Energy Spread

The 60-degree bend magnet shown in figure 3 is used for energy analysis. The analyzer input spot size is set with a quadrupole doublet. This size was typically $1 \mathrm{~cm}$. A fluorescent screen on the analysis focal plane is viewed by a TV camera. The spectrograph dispersion along the screen is $0.6 \mathrm{~cm} / \%$ of energy spread. The screen can measure spreads up to $+5 \%$. The principal measurement error is due to tllagni $\overline{\mathrm{f}}$ ication. A $1-\mathrm{cm}$ input beam spot size appears as $0.18 \mathrm{~cm}$ at the screen (it shrinks a factor of 5), but this still is larger than a zero-size, zero-spread spot. The error that $0.18 \mathrm{~cm}$ represents is $0.3 \%$ energy spread. The measured fullwidth energy spectrum for a $1.2-n C$ micropulse at $10 \mathrm{MeV}$ was $1-1.2 \%$. With the effect of finite beam size considered, the actual spectral spread is estimated to be $0.8-1.0 \%$.

Beam emittance was determined from measurements of spot size as a function of quadrupole lens focal strength. The beam spot sizes were obtained by scanning a 1-mm tungsten wire through the beam and measuring the unscattered current at the spectrometer Faraday cup. A fluorescent screen and TV monitor also provided spot size. The data were computer fit to the expected hyperbolic relationship 6 of beam radius and focal strength. The scan and screen data and an example of the radiusfocal strength $\mathrm{ftt}$ are shown in figure 6 . The hyperbola asymptote is proportional to the beam far field angle, and this together with the minimum radius yield the emittance. The measured emittance for $1.2 \mathrm{nC}$ beam was $0.008 \pm 0.002 \mathrm{~cm}-\mathrm{rad}$.

\section{Conclusion}

The measured single micropulse data at 115 A peak, $1 \%$ energy spread and $0.008 \mathrm{~cm}$-rad emittance will meet the calculated requirements for a high extraction visible FEL oscillator. This injectur design will be directly adapted to a new high voltage linac for that experiment. 
- scar wiae gcatters elfcirons - fatiaday cup after anac yef maginet measuaes pemaining beam - upstream quadpipole aojusts focal gpot size
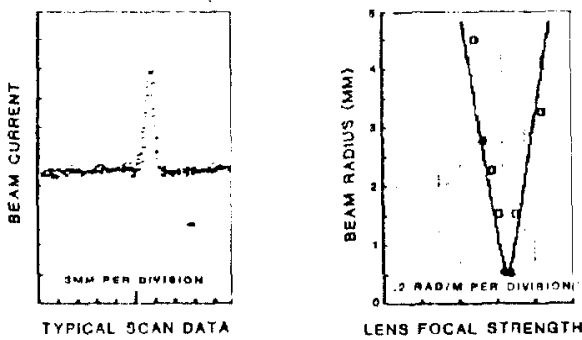

TYPICAL JCAN DATA

LENS FOCAL STMENGTH

- regults $c_{\mathrm{N}}$ =gynth-0080.002 cm.mad

Figure 6. Enit tance Measurement Data

\section{Acknowledgment}

The research leading to this paper was sponsored by Boeing Aerospace Company and DARPA/ONR under contract N00014-82-C-0548.

\section{References}

1. J. L. Adamski, W. J. Fallagher, R. C. Kennedy, B. Kobinson, D. R. Shoffstall, E. L. Tyson, A. M. Vetter and A. D. Yeremian, The Boeing $120 \mathrm{MeV}$ RF Linac for FEL Research, paper E33, this Conference.

2. J. L. Adamski, W. J. Gallagher, R. C. Kennedy and A. D. Yeremian, A High Current Injector for the Boeing Radiation Laboratory FEL Experiment, IEEE Trans. Nuc. Sei., Vo1. NS-30, No. 5, Aug. 1984, p. 2696 .

3. W. B. Hermannsfeldt, Electron Trajectory Program, SLAC Report 226, November 1979.

4. G. W. Peterson and W. J. Gallagher, IEEE Trans. Nuc1. Sci. NS-16, 214 (1969).

5. G. Mavrogenes, et al., Subnanosecond High Intensity Beam Pulse, IEEE Trans. Nuc1. Sci. NS-20, 919 (1973).

6. J. E. Clendenin, G. A. Loew, R. H. Miller, J. L. Pellegrin and $\mathrm{J}$. B. Truher, Single Bunch Beam Measurements for the Proposed SLAC Linear Collider, IEEE Trans. Nuc. Sci., Vo1. NS-28, No. 3, June 1981 . 